\title{
Guidewire Diameter
}

National Cancer Institute

\section{Source}

National Cancer Institute. Guidewire Diameter. NCI Thesaurus. Code C150372.

The diameter of the guidewire. 